\title{
Lactate enhances motility of tumor cells and inhibits monocyte migration and cytokine release
}

\author{
KRISTINA GOETZE ${ }^{1}$, STEFAN WALENTA ${ }^{1}$, MAGDALENA KSIAZKIEWICZ ${ }^{2,3}$, \\ LEONI A. KUNZ-SCHUGHART ${ }^{2}$ and WOLFGANG MUELLER-KLIESER ${ }^{1}$

\begin{abstract}
${ }^{1}$ Institute of Physiology and Pathophysiology, University Medical Center of the Johannes Gutenberg University Mainz, Duesbergweg 6, 55128 Mainz; ${ }^{2}$ Tumor Pathophysiology, OncoRay - Center for Radiation Research in Oncology, Faculty of Medicine Carl Gustav Carus, Dresden University of Technology, Fetscherstr. 74, 01307 Dresden;

${ }^{3}$ Institute of Pathology, University of Regensburg, Franz-Josef-Strauss-Allee 11, 93053 Regensburg, Germany
\end{abstract}

Received February 23, 2011; Accepted April 20, 2011

DOI: $10.3892 /$ ijo.2011.1055

\begin{abstract}
In solid malignant tumors, lactate has been identified as a prognostic parameter for metastasis and overall survival of patients. To investigate the effects of lactate on tumor cell migration, Boyden chamber assays were applied. We could show here that lactate enhances tumor cell motility of head and neck carcinoma cell lines significantly in a dose-dependent manner. The changes in tumor cell migration could be attributed to L-lactate or a conversion of lactate to pyruvate, as only these two substances were able to increase migration. Addition of D-lactate or changes in osmolarity or intracellular $\mathrm{pH}$ did not alter the migratory potential of the cells investigated. Because lactate was shown earlier to impair the penetration of dendritic cells in a tumor spheroid model, which is contrary to the response of the malignant cell population in the present study, we included blood monocytes in our assay as a highly motile immune cell type and precursor of tumor-associated macrophages. Interestingly, high levels of L-lactate $(20 \mathrm{mM})$ at a $\mathrm{pH}$ of 7.4 inhibited monocyte migration in the Boyden chamber system. In addition, cytokine release of TNF and IL-6 was inhibited. The obtained data suggest that high lactate content promotes tumor progression by contributing to the phenomenon of tumor immune escape and by enhancing the migratory potential of the malignant cell population which may directly be coupled to a higher incidence of metastasis.
\end{abstract}

\section{Introduction}

Microenvironment has long been recognized to be important for tumor growth and therapeutic response modification, but

Correspondence to: Dr Kristina Goetze, Institute of Physiology and Pathophysiology, University Medical Center of the Johannes Gutenberg University Mainz, Duesbergweg 6, 55128 Mainz, Germany E-mail: goetze@uni-mainz.de

Key words: cell migration, cytokines, lactate, microenvironment, monocytes, tumor research focused mostly on hypoxia, acidosis and glucose depletion. Little attention was paid to lactate biology, although tumor cells are chronically exposed to largely variable lactate concentrations in vivo. In 1995 and 1997 the first reports appeared demonstrating an interrelationship between the lactate content of primary head and neck tumors and their metastatic spread $(1,2)$. Later on, these data were confirmed not only in head and neck squamous cell carcinomas (3), but also in cervix cancer (4) and in rectal adenocarcinomas (5). The first preliminary observation that a lactate-mediated increase in cancer cell motility might be involved in this correlation was reported by Walenta et al (6), but since then this aspect of cancer biology was not pursued any further until recently.

Research interest in the cell biology of a lactate-enriched environment has been re-vitalized by a recent boom of investigations on carbohydrate metabolism in malignancy and, in particular, on the Warburg effect. Numerous original as well as review articles and special issues of highly ranked journals mirror this development (7-14). The current study has been stimulated by observations on a limited penetration of dendritic cells into multicellular tumor spheroids and their functional inactivation with regard to cytokine release (15). The authors were able to show that these effects were mediated by lactate released from the tumor spheroid cells. Accordingly, tumorderived lactate exerts a similar inhibition on T-lymphocytes in multicellular tumor spheroids (16). From these findings we conclude that a lactate-enriched environment in solid tumors adds to the phenomenon of tumor immune escape by altering the migratory activity and activation potential of defense cells. This may yield a general contribution to the observed reduction in survival of patients with high lactate tumors which is summarized by Walenta et al $(17,18)$. We, however, further hypothesize that the high-lactate environment increases the mobility of cancer cells, an effect that may be causally related to the increased incidence of metastasis in high lactate tumors in the clinic $(17,18)$.

As tumor cells depend upon the Warburg effect to generate energy for proliferation and cell survival, lactate and protons accumulate in these cells $(16,19)$. To maintain a stable intracellular $\mathrm{pH}$, protons are exported into the extracellular space via 
proton extruders or monocarboxylate transporters (MCT). Especially MCT 1 and 4 promote the proton coupled efflux of lactate or pyruvate from tumor cells into the extracellular space, leading to a decrease in extracellular $\mathrm{pH}$ and an increase in lactate concentration (20). The invasion of tumor cells, metastasis and cellular survival as well as tumor progression is influenced by this interstitial acidosis (21). Accordingly, it has been shown that the invasion of melanoma cells in vitro and metastasis in sarcoma and melanoma tumors in vivo is promoted by a decreased extracellular $\mathrm{pH}$ (22). But tumor progression may not only be influenced by the $\mathrm{pH}$ : extracellular lactate could also directly impact cell behavior. As an example, the presence of lactate in the extracellular space upregulates hyaluronan and type I collagen production. In wound healing this generates an environment that enhances migration, an effect which might also hold true for tumor cell migration (20). In addition, a direct link between glycolysis and cell motility was discovered only a few years ago. Glucose-6phosphate isomerase (GPI) a glycolytic enzyme was found to be identical with the autocrine motility factor (AMF). It is overexpressed and secreted in many tumors and is able to stimulate motility in an autocrine manner (reviewed in ref. 17).

In the present investigation the hypothesis of exogenous lactate to directly affect cell motility was challenged by studying the migratory activity of tumor cells and blood monocytes in high lactate environments in vitro. The Boyden chamber technique was used for the quantification of cell motility. One major focus of the study was to validate the specificity of lactate-induced tumor cell migration. This was done by including various control conditions that for example demonstrated a pro-migratory effect of L-lactate but not D-lactate on tumor cells.

\section{Materials and methods}

Cell lines and culturing conditions. The association of a lactate-enriched microenvironment and decreased patient survival was most spectacular in squamous cell carcinomas of the head and neck and, less striking but statistically significant, in cervix tumors $(17,18)$. The majority of experiments was therefore performed with human SQ20B cells which were derived from squamous carcinoma of the human larynx $(23,24)$. These cells were cultured in Dulbecco's modified Eagle's medium: nutrient mixture F12 (DMEM/F12; Gibco Life Technologies, Karlsruhe, Germany) supplemented with $20 \%$ Cosmic calf serum (Hyclone, Logan, UT, USA), $1 \%$ penicillin/streptomycin and $0.4 \mu \mathrm{g} / \mathrm{ml}$ hydrocortisone (SigmaAldrich, Taufkirchen, Germany) using an incubator (Flow Laboratories, Irvine, UK) with a humidified atmosphere at $37^{\circ} \mathrm{C}$ with $5 \% \mathrm{CO}_{2}$ and air. To exclude cell line specificity of the effects registered, migration experiments were expanded to include PCI13 cells that were derived from a squamous cell carcinoma of the retromolar trigonum (25). To include cells derived from a tumor entity other than head and neck cancer, the migratory effect of lactate was additionally investigated using SiHa cells which were derived from a cervix carcinoma (26). Cells from all three cell lines were routinely transferred by trypsinization and re-seeding according to a standardized protocol for up to 20 passages, after which they were replaced by cells from a frozen stock.
Monocyte isolation. Human monocytes were isolated from blood of healthy donors by leukapheresis. This was followed by a ficoll/hypaque density gradient centrifugation and separation with counter-current centrifugation (centrifuge: J6M-E, Beckmann, Munich, Germany) (27-30). Purity of monocytes was tested via flow cytometry and immune phenotyping taking into account the expression of the monocyte-specific antigen CD14 (31). Isolated cell populations were only used for further studies when the proportion of CD14 expressing cells (monocytes) was $>85 \%$.

Migration assay with tumor cells. Commercial 48-well chemotaxis chambers (NeuroProbe, Gaithersburg, MD, USA) were used for a classical Boyden chamber assay. The chemotaxis chamber consists of two Lucite slides each with 48 cylindrical holes. The holes in the bottom slide represent wells that can be readily filled with $30 \mu \mathrm{l}$ fluid media. The slide is then covered with a $25-\mu \mathrm{m}$ thick polycarbonate membrane with $8-\mu \mathrm{m}$ pores which is coated with gelatin at its bottom and collagen I at its upper side. After the adjustment and fixation of the lid on the polycarbonate membrane and bottom slide by appropriate screws, $50 \mu \mathrm{l}$ cell suspensions can be pipetted into the lid holes and the whole sandwich chamber is transferred to the incubator to start the migration assay. In the present investigation, each starting compartment was filled with a suspension of $2.5 \times 10^{5}$ cells $/ \mathrm{ml}$. In some experiments chemical modifiers of cell motility were added to some of the 48 wells, and the effect on cell migration was evaluated by comparing averages from stimulated versus control wells, with 8-24 evaluated wells per condition. All migration studies were performed in 3-5 independent experiments under standard incubator conditions. Chambers were incubated for various observation periods ranging from 3 to $20 \mathrm{~h}$ with the majority of the measurements done with an incubation time of $16 \mathrm{~h}$. After termination of the migration experiment and disassembly of the chamber, cells attached to the upper side of the polycarbonate membrane were removed, and the remaining cells in the gelatin were chemically fixed with methanol and stained with Hemacolor staining kit (Merck, Darmstadt, Germany). Cells were counted in seven visual fields in all wells in a microscope (magnification, x400; Leica DM IRB, Leica, Bensheim, Germany).

Biochemical modification of tumor cell migration. The major focus of the study was the cell biology of a tumor-like microenvironment with regard to the accumulation of tumor cell-derived lactate. The average tissue content of lactate in solid tumors has been measured to exceed $15 \mu \mathrm{mol} / \mathrm{g}$ reaching values of $40 \mu \mathrm{mol} / \mathrm{g}$ and even higher within selective tumor regions (4). Converting these values to equivalent concentration units under consideration of the high water content of tumor tissue, the values can be approximated by 15 and $40 \mathrm{mM}$, respectively. L-lactate, D-lactate, pyruvate, sodium chloride $(\mathrm{NaCl})$ or amiloride (5-(N,N-dimethyl)amiloride hydrochloride) were used as chemical modifiers. The concentrations given for lactate and pyruvate are final concentrations in the medium and were verified using standard photometrical assays. The concentration given for sodium chloride was added to the medium originally containing $120.6 \mathrm{mM}$ $\mathrm{NaCl}$. 
Time-lapsed video microscopy of tumor cell motility. Images of migrating SQ20B cell clusters were acquired every $10 \mathrm{~min}$ over an observation period of 510 min with a special video system. Therefore cells were seeded in standard culture dishes prior to observation and cell culture medium was added with or without the addition of $20 \mathrm{mM}$ L-lactate.

Migration assay with monocytes. Monocyte migration was also determined in the 48-well chemotaxis chambers, as described above for tumor cells. In this setting a polycarbonate membrane with $5 \mu \mathrm{m}$ pores (Osmonics Inc., Minnetonka, MN, USA) was used and the holes in the bottom slides were filled with $30 \mu \mathrm{l}$ of DMEM with $1 \mathrm{~g} / 1$ glucose, $10 \%$ fetal calf serum, $100 \mathrm{U} / \mathrm{ml}$ penicillin and $100 \mu \mathrm{g} / \mathrm{ml}$ streptomycin (all from PAN Biotech, Aidenbach, Germany). In every experimental series the addition of 0.01 and $1 \mu \mathrm{M}$ N-formyl-L-methionylleucyl-L-phenylalanine (FMLP; Sigma-Aldrich) in DMEM in the wells in the bottom chamber served as positive and unconditioned DMEM as negative control. Each well of the upper chamber was filled with $5 \times 10^{4}$ monocytes in $50 \mu \mathrm{l}$ freshly supplemented DMEM. The migration chamber was then incubated for $2 \mathrm{~h}$ under standard culture conditions. After careful removal of non-migrated cells, membranes were air-dried, fixed in $70 \%$ methanol and stained as depicted earlier. After transferring the membranes onto glass slides, migrated monocytes were counted in a microscope. Four high-power fields (magnification, $x 400$ ) were evaluated per well. A minimum of three wells per condition was analyzed. Experiments were repeated at least three times, i.e. with monocytes from a minimum of three different healthy donors, and the mean numbers of migrated monocytes \pm intraexperimental standard deviations were calculated. To test the influence of L-lactate on monocyte migration $2-20 \mathrm{mM}$ lactate with a $\mathrm{pH}$ adjusted to $7.4 \pm 0.1$ were added in the wells of the lower chamber as indicated (see Results).

Monocyte activation assay. The activation capacity of monocytes in different lactate concentrations was characterized using the cytokine release of TNF and IL- 6 in media supernatant as an indicator. For this approach, $1 \times 10^{6}$ monocytes in $1 \mathrm{ml}$ serum-free RPMI were seeded into each well of 6-well culture plates and incubated under standard culture conditions. After 20-30 min $1 \mathrm{ml}$ RPMI containing 4\% human AB serum and antibiotics (PAN Biotech) with and without lactate adjusted to a $\mathrm{pH}$ of $7.4 \pm 0.1$ was added to reach a final serum concentration of $2 \%$ and the various lactate concentrations of interest $(2-20 \mathrm{mM})$. The commercial RPMI used for these experiments was certified LPS-free. Both, LPS-stimulated and unstimulated monocytes were studied. LPS was added in parallel to serum and lactate to obtain $100 \mathrm{ng} / \mathrm{ml}$. Monocytes from donors with a high stimulation status prior to LPS addition and cells which could not be activated with LPS were not taken into account. The cytokine release after $16 \mathrm{~h}$ was detected by measuring cytokine levels in supernatants with a standardized ELISA kit (R\&D Systems, Wiesbaden, Germany). The readout was performed in a commercial ELISA reader (Emax, Molecular Devices, Sunnyvale, CA, USA). The acquired data were documented in absolute values and relative to the donor-specific LPS-stimulated controls set as $100 \%$.
Monocyte cell viability/survival assessment. Three approaches were used to evaluate cell survival in a lactate-enriched environment. a) Density and viability of adherent monocytes in 6-well plates applied for activation studies as described above were routinely monitored and imaged after $16 \mathrm{~h}$ of incubation using an inverted phase-contrast microscope (Axiovert200, Carl Zeiss, Goettingen, Germany) combined with a digital camera and software system. Here, trypan blue was added at a final concentration of $0.05 \%$ in PBS for $10 \mathrm{~min}$ to identify potential lactate-induced cell destruction with 1,000 adherent cells being counted in phase contrast images. b) In an independent experimental series, $2 \times 10^{5}$ monocytes in $0.5 \mathrm{ml}$ serum-free DMEM were inoculated in 24-well plates. After $30 \mathrm{~min}$ of incubation, freshly prepared lactate solutions in DMEM with and without FCS were added to reach final concentrations of $20 \mathrm{mM}$ and $10 \%$, respectively. The number of adherent cells was then monitored after an additional $1.5 \mathrm{~h}$ incubation interval by counting the number of adherent cells in five high-power field images (objective, x40) in each of three wells per condition. c) In a third experimental set-up, aliquots of $1 \times 10^{6}$ freshly isolated monocytes in $5 \mathrm{ml}$ DMEM containing $10 \%$ FCS supplemented with or without exogenous lactate (up to $40 \mathrm{mM}$ ) were transferred into Teflon bags, sealed and incubated as suspension cultures for $4 \mathrm{~h}$ under standard culture conditions. Monocytes were then harvested and cell counts and volumes were recorded with a Casyl cell analyzer system (Schaerfe System/Innovatis, Reutlingen, Germany) which was also used for routine culture quality assessment. In parallel, harvested cells were examined with the trypan blue exclusion method for validation (final concentration: $0.03 \%$ trypan blue, 2 min). Experimental set-ups a) and c) were performed with monocytes from three independent donors; approach b) was carried out with monocytes from one donor only. All lactate exposure experiments were at a $\mathrm{pH}$ adjusted to $7.4 \pm 0.1$.

Western blotting. For protein analysis a standard semi-dry Western blotting was used. Cells were lysed and $10 \mu \mathrm{g}$ protein were applied on a $8 \%$ SDS polyacrylamide gel. After electrophoresis the proteins were blotted on a PVDF membrane following antibody-coupled protein detection. $\beta_{1}$ integrins were detected using monoclonal mouse anti-human CD29 anti-bodies, (Biogenex, San Ramon, CA, USA) and antimouse IgG peroxidase-conjugated antibodies (Sigma, Deisenhofen, Germany). The phosphorylated $\beta_{1}$ integrins were detected using rabbit polyclonal anti-integrin $\beta_{1}$ receptor [pTpT $^{788 / 789}$ ] phosphospecific antibodies (BioSource International, Camarillo, CA, USA) and anti-rabbit IgG peroxidase conjugated antibodies (Santa Cruz, Heidelberg, Germany). Actin was used as a housekeeping protein and was detected with goat anti-human actin antibodies (I-19) and donkey anti-goat IgG peroxidase-conjugated antibodies (both Santa Cruz). Binding of the peroxidase-conjugated antibodies was verified on film using an enhanced chemiluminescence kit (ECLKit; Amersham Biosciences, Buckinghamshire, Great Britain). For computer-based analysis of the $\beta_{1}$ integrin expression and phosphorylation the optical densities of the detected bands were determined with ImageJ, corrected for background and normalized to the optical densities of the corresponding actin bands. 
A

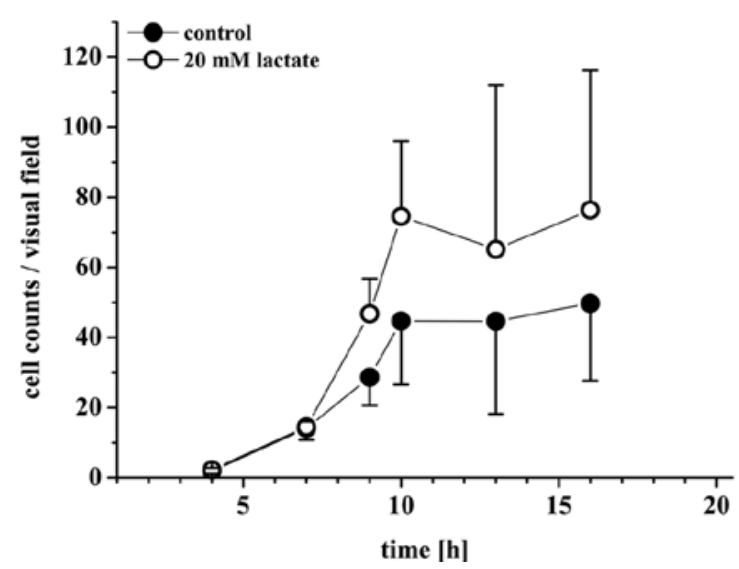

B

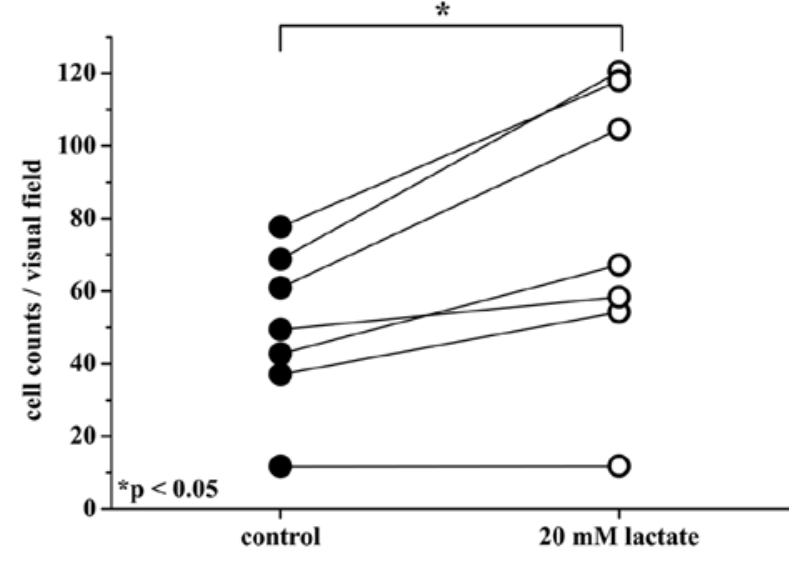

Figure 1. Kinetics and statistical significance of SQ20B tumor cell migration under the influence of lactate. (A) The migration of SQ20B cells under the influence of lactate was investigated in Boyden chamber assays as paired experiments at several time points over a period of $19 \mathrm{~h}$. The mean cell counts per visual field were plotted as a function of time showing the mean \pm SD for three to seven independent experiments (with one chamber consisting of at least 8 single wells which were evaluated). (B) As an example for the statistical significance and variation of the observations the mean cell counts per visual field for seven independent experiments with an incubation period of $16 \mathrm{~h}$ are shown for SQ20B cells with or without $20 \mathrm{mM}$ lactate. Significant differences between treated and untreated cells: "p<0.05, t-test. Black dots, untreated SQ20B control; black circles, SQ20B cells + 20 mM L-lactate.

\section{Results}

Lactate-induced enhancement of cell motility. Tumor cell migration studied in Boyden chamber assays underlie a kinetic which starts with an initial increase in the number of migrated cells overtime and then reaches a plateau. With SQ20B cells which were studied here, this plateau is reached after approximately $10 \mathrm{~h}$. To test if this kinetic is also valid under addition of exogenous L-lactate, migration of SQ20B cells was monitored in Boyden chamber assays over $19 \mathrm{~h}$ in paired experiments. In this case one chamber was used to simultaneously observe the migration under control condition as well as under the addition of $20 \mathrm{mM}$ L-lactate. At every given point $n=3-7$ independent paired experiments were performed. The mean cell number per visual field $( \pm$ SD) as a function of incubation time is shown in Fig. 1A. It becomes obvious in this plotting that the migration of SQ20B cells follows the same kinetics under both conditions. However, the mean number of migrated cells is constantly higher in the $20 \mathrm{mM}$ lactate medium compared to the controls at any time point studied. Because of high inter-experimental variations in the migrated tumor cells per visual field reflected by the standard deviation in Fig. 1A, B representatively documents the result of seven independent paired assays analyzed at one time point (incubation period of $16 \mathrm{~h}$ ). Here, the data from each paired observation are connected with a line to visualize that the number of migrated cells is consistently enhanced in the lactate-conditioned environment as compared to the corresponding untreated control. The lactate-induced increase in tumor cell migration is significant $(\mathrm{p}<0.05)$.

For standardization of further experiments a uniform incubation time of $16 \mathrm{~h}$ was chosen to guarantee that all data represent endpoint results with regard to the migration kinetics in the Boyden chamber. To also exclude a potential tumor cell line-specificity of the observed effect, cell migration after the addition of $20 \mathrm{mM}$ lactate was also analyzed for the tumor cell lines PCI13 and SiHa in Boyden chamber assays. These cells showed similar results with an increase in tumor cell migration after the addition of lactate (data not shown).

Since the first results indicated migratory changes in SQ20B cells after the addition of exogenous L-lactate, we wanted to investigate, if this effect is concentration-dependent. Therefore, Boyden chamber assays were performed with the addition of 10, 20, 30 and $40 \mathrm{mM}$ L-lactate. Fig. 2A displays the mean relative cell migration of 4-5 independent experiments $( \pm$ SD) as a function of dose. Control cells (without the addition of L-lactate) were set at $100 \%$ (dotted line). It could be shown that the addition of lactate significantly enhances the motility of SQ20B cells in a concentration-dependent manner. Tumor cell migration of these cells is increased to up to $170 \%$ compared to untreated controls reaching a plateau after the addition of 30-40 $\mathrm{mM}$ lactate.

Since SQ20B cells tend to form clusters of cells on adhesive substrates, and since cohort migration of tumor cells may be distinctly different from single cell migration (as observed in Boyden chamber assays) (32), the cluster motility in culture dishes was monitored using a special video system. As the most significant changes in the Boyden chamber assays became apparent after the addition of $20 \mathrm{mM}$ lactate ( $\mathrm{p}<0.005$, t-test), this concentration was used in the time lapse video microscope experiments. SQ20B cells were seeded in common culture dishes and the migration of cell clusters was imaged by taking microphotographs every $10 \mathrm{~min}$ for $510 \mathrm{~min}$. Representative recordings of migrating cells are displayed in Fig. 2B with black and white circles marking migrating cell clusters. In contrast to cells incubated without lactate, intensive clustered cell migration was observed by the addition of $20 \mathrm{mM}$ lactate. These results suggested that a lactate-enriched environment not only enhances single-cell migration but also shifts the mode of migration from single-cell motion to cluster formation and migration.

Specificity of lactate-enhanced tumor cell migration. All results in Figs. 1 and 2 were obtained by adding L-lactate, 
A

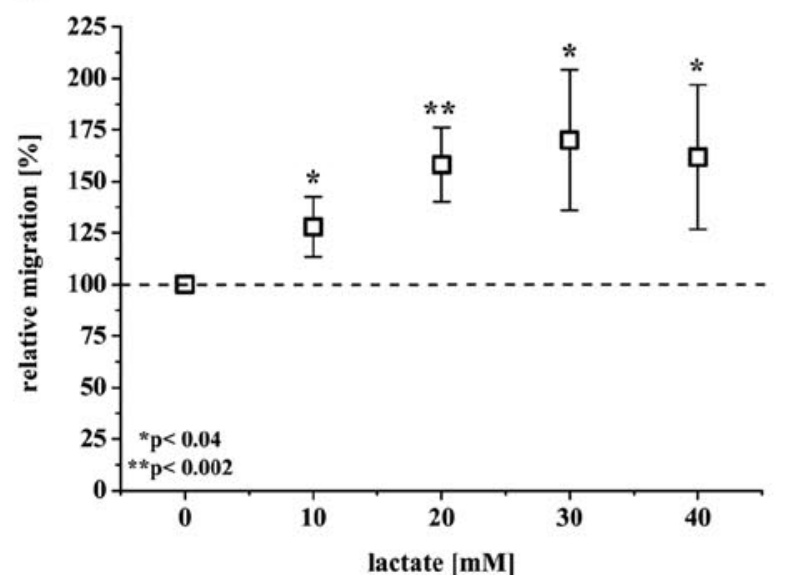

B

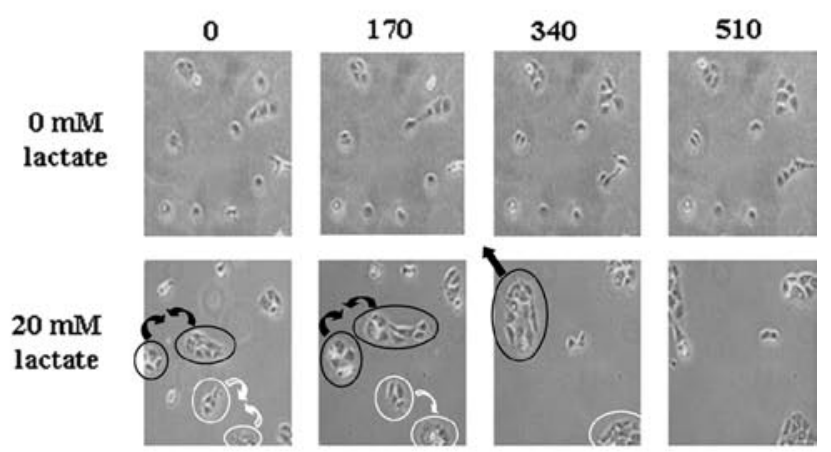

Figure 2. Migration of SQ20B cells under the influence of L-lactate. (A) Boyden chamber assay over $16 \mathrm{~h}$ with SQ20B tumor cells with media supplemented with $0,10,20,30$ and $40 \mathrm{mM}$ L-lactate. The relative mean numbers of migrated cells $( \pm \mathrm{SD})$ of at least three independent experiments from each of three independent researchers were plotted against the lactate concentration with the migration of untreated cells set at $100 \%$. Significant differences between treated and untreated cells: ${ }^{*} \mathrm{p}<0.05,{ }^{* *} \mathrm{p}<0.005$, t-test. (B) Time lapse video microscopy of migrating SQ20B cells treated with $20 \mathrm{mM}$ L-lactate. Cells were seeded with and without $20 \mathrm{mM}$ lactate in a culture dish, and migration of cell clusters were observed by snapshots every 10 min for 510 min. Two different cell clusters are marked with black and white lines. These clusters enlarge and fuse to form one large cluster that migrates leaving the visual field.
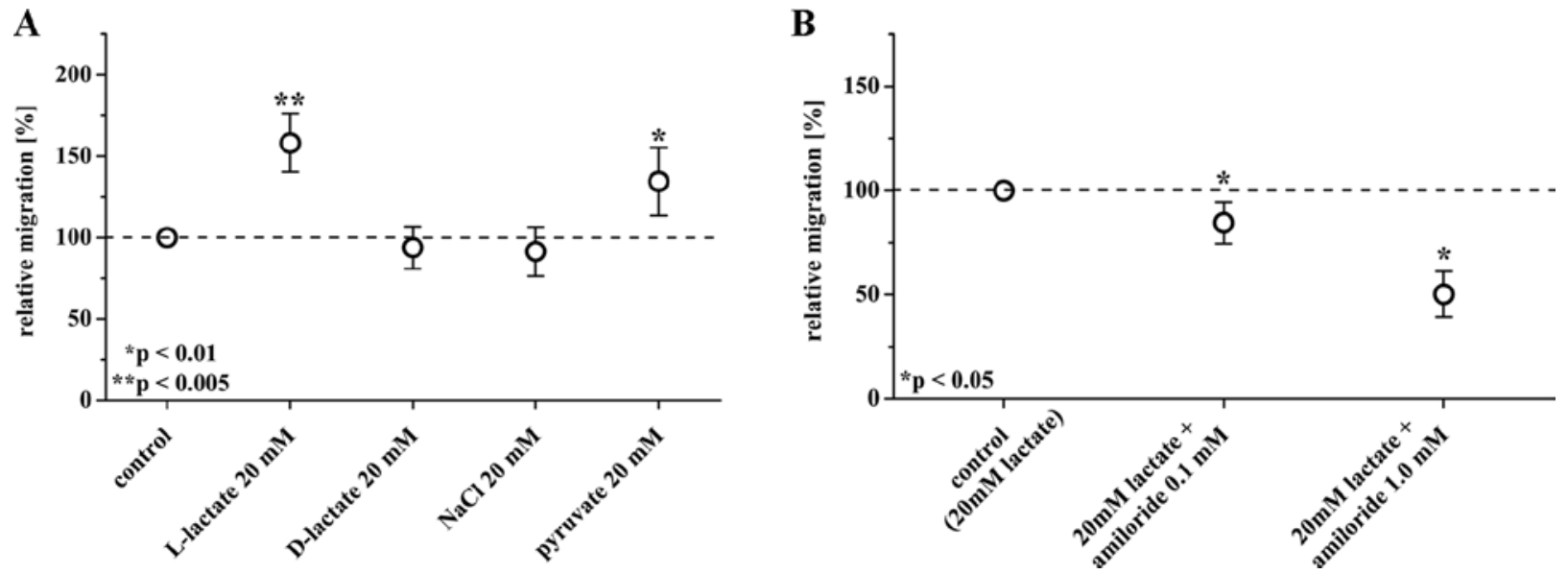

Figure 3. Specificity of the effect of L-lactate on tumor cell migration in a Boyden chamber assay. Cell migration of SQ20B cells was observed in standardized Boyden chamber assays after the addition of (A) L-lactate $(20 \mathrm{mM})$, D-lactate $(20 \mathrm{mM})$, sodium chloride $(\mathrm{NaCl}, 20 \mathrm{mM})$ or pyruvate $(20 \mathrm{mM})$. The mean relative migration $( \pm \mathrm{SD})$ from 5-12 independent experiments is shown with untreated control cells set as $100 \%$. Significant differences compared to untreated control cells: " $\mathrm{p}<0.01$, t-test. (B) The cell migration of SQ20B cells treated with $20 \mathrm{mM} \mathrm{L}$-lactate was set as control at $100 \%$ for comparing the influence of media additionally supplemented with amiloride at concentrations of 0.1 and $1.0 \mathrm{mM}$. Mean relative migration $( \pm \mathrm{SD})$ from seven independent experiments is shown. ${ }^{*} \mathrm{p}<0.05$, t-test.

which is a product of glucose metabolism in most cancer cells, and which chronically accumulates in such high concentrations in solid tumors. To verify that the observed migratory changes could exclusively be attributed to a variation of the lactate concentration and not to an unspecific cellular response, such as metabolic activation, a number of control experiments were carried out. The migratory effects induced by the addition of $20 \mathrm{mM}$ D-lactate, $20 \mathrm{mM} \mathrm{NaCl}$, or $20 \mathrm{mM}$ pyruvate are displayed in Fig. 3A as mean relative migration ( \pm SD) compared to untreated control cells (set as 100\%, dotted line).

At first, the enantiomer D-lactate was used to check for the biochemical specificity of the migratory stimulation. The addition of $20 \mathrm{mM}$ D-lactate did not change the cell migration of SQ20B cells significantly, as results obtained were close to those using untreated control cells. The same cell line was analyzed with regard to osmotic effects. After adding $20 \mathrm{mM}$ lactate, $\mathrm{pH}$-changes of the culture medium were neutralized by the addition of $\mathrm{NaOH}$. This raised the osmolarity of the medium to up to $40 \mathrm{mosmol} / \mathrm{l}$. To test for a possible influence of osmotic effects on cell motility and to verify that the changes in cell migration could not be attributed to the changed osmolarity $20 \mathrm{mM}$ sodium chloride $(\mathrm{NaCl})$ were added instead of lactate. Similarly as with D-lactate, no changes in migration were observed under these conditions. Only the addition of $20 \mathrm{mM}$ pyruvate promoted the migration of SQ20B cells significantly $(\mathrm{p}<0.01)$ to $134 \%$ compared to untreated cells and, thus, could in part mimic the results obtained after addition of $20 \mathrm{mM}$ L-lactate. 
If extracellular lactate concentrations are elevated, an intracellular acidification cannot be entirely ruled out even at a clamped extracellular $\mathrm{pH}$. Even if the buffer capacity of the culture medium is relatively high and the $\mathrm{pH}$ was adjusted to 7.3 , it is possible that lactate together with protons is taken up via the MCTs of these cells. This would induce a decrease in the intracellular $\mathrm{pH}$ which might also influence tumor cell migration. To test if the addition of lactate is linked to intracellular acidification, the $\mathrm{H}^{+}$-export was inhibited by the addition of amiloride, an inhibitor of the sodium/proton antiport (33). In this experimental set up media were supplemented with two different amiloride concentrations ( 0.1 and $1.0 \mathrm{mM}$ ) in addition to adding $20 \mathrm{mM}$ L-lactate. The induced migration of SQ20B tumor cells could be inhibited significantly $(\mathrm{p}<0.05)$ by adding amiloride in a dose-dependent manner (Fig. 3B).

Effect of lactate gradients on monocyte migration. The experimental series with tumor cells revealed that lactate enhances tumor cell migration. It has been described earlier, however, that the migration of some immune cell types, i.e. dendritic cells, is inhibited by lactic acid in a spheroid coculture model (15). In order to further verify our finding in tumor cells, we included blood monocytes in our study as a very rapid and motile control cell type. These cells are recruited to tumor sites where they undergo differentiation processes often leading to an abnormal tumor-associated phenotype that contributed to tumor angiogenesis and progression. To exclude the $\mathrm{pH}$ effect, we particularly analyzed the impact of lactate in a $\mathrm{pH}$ re-adjusted environment on the migratory activity of blood monocytes in a standardized Boyden chamber assays as well as their activation capacity.

Fig. 4 shows the mean relative migration $( \pm \mathrm{SD})$ of monocytes after the addition of different lactate concentrations to the lower chamber resulting in a lactate gradient. Migration of untreated cells (labeled as medium control) was taken as control and set as $100 \%$. The addition of FMLP to the lower chamber served as positive control (labeled FMLP control-high) for the donor-dependent activation of monocyte migration. Cells that could not be induced to migrate towards high FMLP concentrations were excluded from further analyses. Overall, the experiments demonstrated a high donor dependency of migration to elevated lactate levels. Some donor monocytes showed higher migratory activity towards $2-5$ or $10 \mathrm{mM}$ lactate compared to untreated cells while others remained rather unaffected under these conditions or showed a reduced migratory activity at higher concentrations. In summary, there was no consistent change in monocyte migration towards lactate concentrations of up to $10 \mathrm{mM}$ in a $\mathrm{pH}$ neutralized environment. However, migration was reproducibly absent for all donor monocytes, if $20 \mathrm{mM}$ lactate was applied.

The absence of migration after the addition of $20 \mathrm{mM}$ lactate to the lower chamber in the Boyden assay might indicate that $20 \mathrm{mM}$ affects monocyte viability. This, however, was not the case as verified by various experimental settings using adherent and suspension monocyte cultures with human, fetal calf or without serum supplementation incubated with and without lactate up to $20 \mathrm{mM}$ at a $\mathrm{pH}$ of $7.4 \pm 0.1$. As an example, after $1.5 \mathrm{~h}$ of exposure the average number of adherent cells in five high-power field images of three wells of

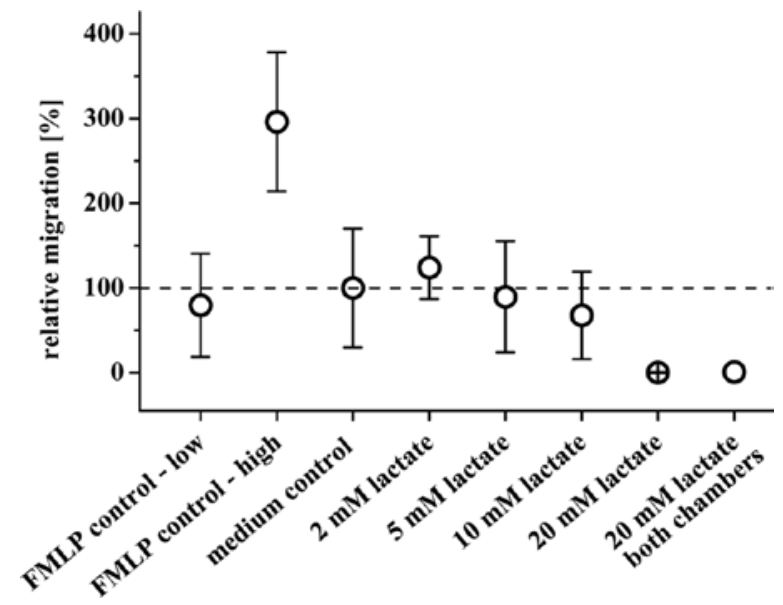

Figure 4. Monocyte migration after the addition of exogenous lactate. Freshly isolated monocytes were incubated for $2 \mathrm{~h}$ in a Boyden chamber assay. The mean relative monocyte migration $( \pm \mathrm{SD})$ from three independent experiments (monocytes from three healthy donors) was plotted against the different lactate concentrations. The mean relative migration of monocytes in untreated media (labeled medium control) were taken as control and set as $100 \%$. FMLP was used to test whether the isolated monocytes could be activated to migrate (FMLP control - high).

a 24-well plate after seeding of $2 \times 10^{5}$ monocytes was $1057 \pm 177$ monocytes in DMEM without FCS, 1256 \pm 44 in DMEM with $10 \%$ FCS, $1217 \pm 44$ in DMEM without FCS supplemented with $20 \mathrm{mM}$ lactate and $1103 \pm 25$ when incubated with DMEM supplemented with both $10 \%$ FCS and $20 \mathrm{mM}$ lactate. After $16 \mathrm{~h}$ of exposure to $0,2,5,10$ or $20 \mathrm{mM}$ L-lactate, the proportion of trypan blue-positive cells was 3-4\% in all LPSfree adherent cultures from three independent donors. The addition of LPS resulted in a slight reduction in cell viability but lactate exposure (up to $20 \mathrm{mM}$ ) did not further increase the mean trypan blue-positive fraction of $11-12 \%$. The recovery of monocytes from Teflon bag suspension cultures was $98-100 \%$ when exposed to up to $20 \mathrm{mM}$ lactate for $4 \mathrm{~h}$. In this approach, we also applied $40 \mathrm{mM}$ L-lactate at a $\mathrm{pH}$ re-adjusted to $7.4 \pm 0.1$ and found monocyte survival to be reduced to $48 \pm 6 \%$ for monocytes from three independent donors under such high lactate concentrations.

Activation status of monocytes after addition of lactate. To test, if the increase in lactate concentration not only affects the migration of monocytes but also their activation capacity, the release of IL-6 and TNF was measured. For this purpose, inactivated and LPS-activated monocytes were incubated for $16 \mathrm{~h}$ with and without lactate, and cytokine release was quantified in the supernatant via ELISA. Fig. 5 documents the corresponding results [IL-6 (A); TNF (B)] showing the mean cytokine release relative to LPS-activated monocytes without lactate exposure which were set as $100 \%$ (LPS-stimulated w/o). No clear change in cytokine release was detected in unstimulated monocytes. However, cells that were activated with LPS revealed considerable alterations in IL-6 and TNF release following lactate exposure. While the addition of 2 and $5 \mathrm{mM}$ lactate did not significantly and/or consistently modified the mean cytokine release of stimulated monocytes from four healthy donors, cytokine concentrations in the supernatants were reduced when these monocytes were exposed 
A

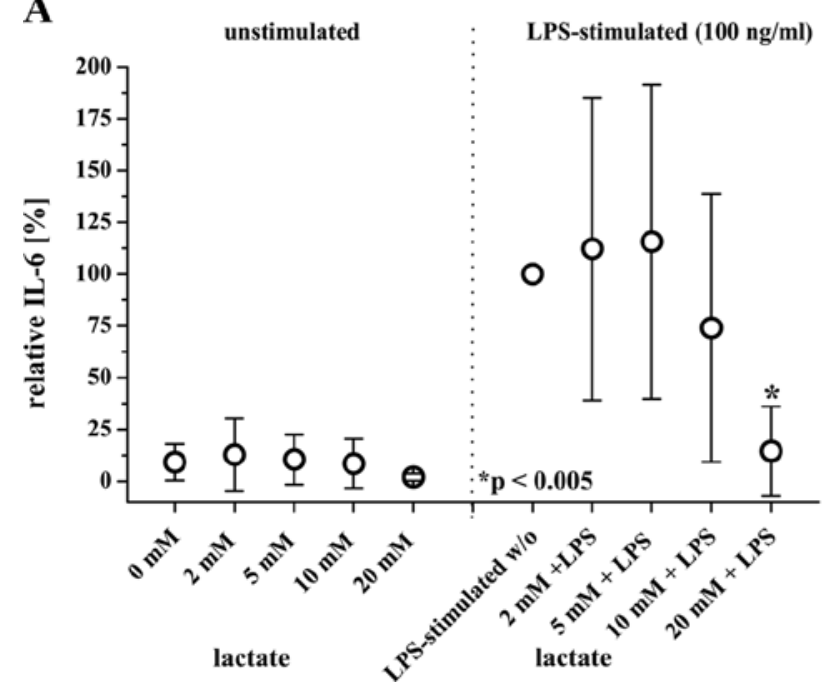

B

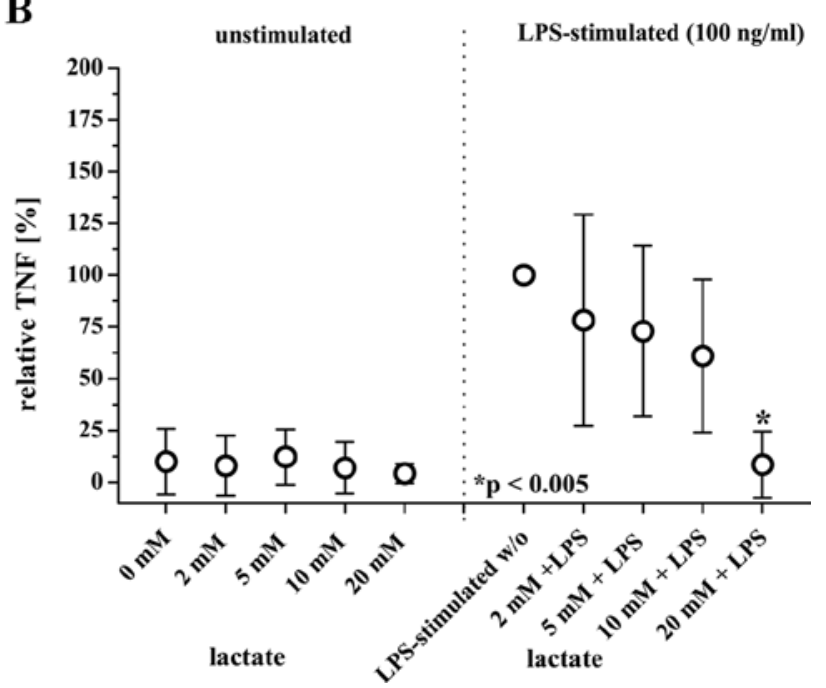

Figure 5. Cytokine release of monocytes with and without lactate. Monocyte activation was measured by the release of IL-6 (A) and TNF (B) in media supernatant after $16 \mathrm{~h}$ with and without L-lactate. Mean cytokine release ( \pm SD) of four independent experiments, i.e. with monocytes from four healthy donors are shown relative to the respective LPS-stimulated monocyte control (labeled LPS-stimulated w/o) set as $100 \%$. $^{*}$ p $<0.005$.

to 10 or $20 \mathrm{mM}$ lactate. Lactate $(20 \mathrm{mM})$ resulted in IL-6 and TNF signals significantly $(\mathrm{p}<0.005)$ lowered to 14 and $8 \%$, respectively, of the related lactate-free controls.

$\beta_{1}$ integrin expression and L-lactate. Tumor cell and monocyte migration depend upon cellular interaction with the extracellular matrix. One important group of proteins mediating this is the transmembrane glycoprotein family of integrins. These heterodimeric proteins interact with different components of the extracellular matrix, e.g. collagen, control cytoskeletal organization and thus play a vital role in cell adhesion, invasion and migration (34). It has been known for quite some time, that the expression and activation of the $\beta_{1}$ integrin domain influences tumor cell migration and invasion $(35,36)$. We were therefore interested in the expression pattern of this integrin and its potential influence on lactate-induced tumor cell migration. Thus SQ20B cells were incubated for $16 \mathrm{~h}$ (= time of Boyden chamber experiment) with $20 \mathrm{mM}$ L-lactate and lysed for Western blot analysis. As an example, representative Western blots for the expression $\left(\beta_{1}\right.$, left panel) and phosphorylation ( $\mathrm{p} \beta_{1}$, right panel) of $\beta_{1}$ integrin and the corresponding expression of the detected housekeeping protein actin are shown in Fig. 6A. For further evaluation the optical density of these obtained bands in six independent experiments were measured and normalized to the expression of actin. These data as well as the mean results ( \pm SD) are plotted in Fig. 6C with corresponding samples depicted as similar icons. As documented, the incubation with lactate affects the expression and phosphorylation of $\beta_{1}$ integrins in tumor cells differently. Although the expression level of $\beta_{1}$ integrin is raised by lactate exposure $(\mathrm{p}<0.01)$ its phosphorylation decreased significantly. This suggests that the addition of L-lactate favors the expression of $\beta_{1}$ integrin in tumor cells, but not the phosphorylation of this protein at the tyrosin residues 788 and 789 .

To also test for the importance of $\beta_{1}$ integrin expression and phosphorylation on monocytes, we incubated monocytes in Teflon bag suspension culture with given concentrations of lactate for $4 \mathrm{~h}$ and also analyzed the cell lysates of $1 \times 10^{7}$ cells in Western blot experiments. In monocytes, integrin expression in general was very low and in four out of six independent monocyte preparations under the detection level in our Western blot assay when up to $50 \mu \mathrm{g}$ protein was loaded onto the polyacrylamide gel. Integrin was detectable in two monocyte preparations, but no clear difference between control and $20 \mathrm{mM}$ lactate-treated monocytes could be detected. Also, no clear and consistent alteration induced by the addition of lactate could be observed in $\beta_{1}$ integrin phosphorylation (Fig. 6B and D).

\section{Discussion}

Glycolysis has been claimed earlier as primary energy source in tumor cell chemotaxis. Most observations, however, relate to genetic alterations in metabolic pathways accompanying tumor cell progression while we studied tumor cell migration rather as an epigenetic phenomenon induced by local lactate levels. It is recognized that the microenvironmental situation in tumors is much more complex with locoregional and spatiotemporal variations in oxygen, glucose, lactate, $\mathrm{pH}$, carbon dioxide and many other factors. It is a sophisticated task to mimic the tumor microenvironment in vitro in a comprehensive manner for mechanistic studies. Such complex approaches require insight into the role of each individual factor on the parameter of interest such as migratory activity. Because lactate seems a robust endpoint of tumor micromilieu beyond hypoxia that mirrors many biological and clinically relevant characteristics, our study has focused on this metabolite. The lactate content of primary malignant lesions has been identified as a prognostic parameter for metastasis and overall survival of patients. It could be shown in different clinical studies that a high amount of lactate is correlated with increased propensity to early metastasis formation and poor prognosis for overall survival (17). We therefore hypothesized that a lactateenriched environment promotes the mobility of cancer cells 
A

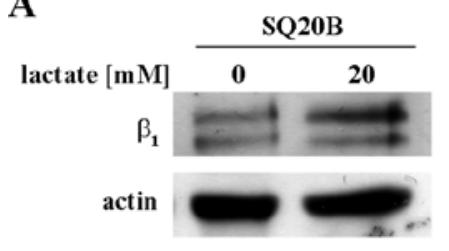

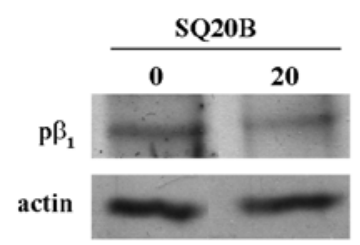

C

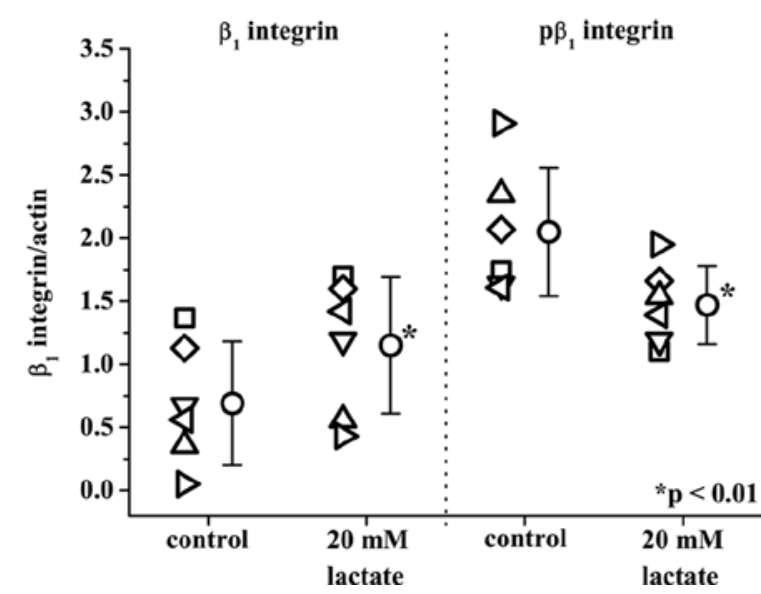

B
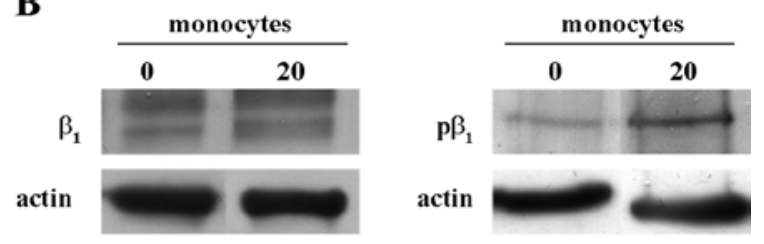

D

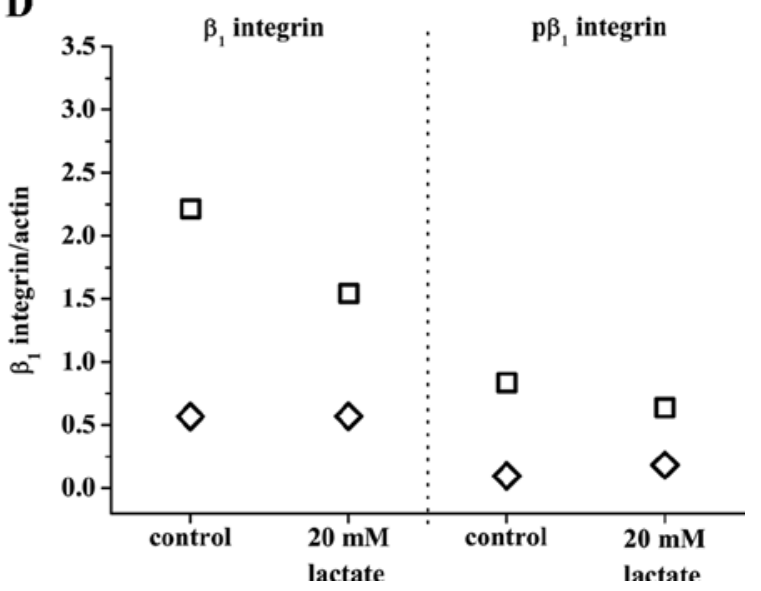

Figure 6. $\beta_{1}$ integrin expression and activation in SQ20B cells and monocytes incubated with $20 \mathrm{mM}$ L-lactate. The expression and phosphorylation of $\beta_{1}$ integrin was tested in Western blot experiments in SQ20B cell (A and C) and monocyte lysates (B and D). SQ20B cells were incubated for $16 \mathrm{~h}$ with $20 \mathrm{mM}$ lactate, lysed and $10 \mu \mathrm{g}$ protein were applied in Western blot experiments. (A and B) Representative Western blotting is shown for the expression (left panel) and activation (right panel) of $\beta_{1}$ integrin and the expression of the corresponding housekeeping protein actin. (C and D) For analysis the optical densities of the detected bands were measured and the integrin expression was normalized to actin. The results obtained are plotted as $\beta_{1}$ integrin expression/actin expression for untreated control cells and for cells incubated with $20 \mathrm{mM}$ lactate. $(\mathrm{C})$ The results of six independent experiments as well as the mean $( \pm$ SD) are displayed for SQ20B cells. Corresponding results are marked with similar symbols. Significant differences between treated cells and control cells: ${ }^{*} \mathrm{p}<0.01$. (D) The results of two independent Western blottings of $\beta_{1}$ integrin expression and activation in monocytes are shown with the corresponding results marked with similar symbols.

contributing to the above mentioned correlation of lactate content and metastasis incidence in solid tumors.

The present study clearly shows that tumor cell migration in head and neck carcinoma cell lines in vitro is significantly increased by the addition of exogenous L-lactate in a dosedependent manner. This enhancement in migratory activity is not tumor cell line-dependent and was specific for L-lactate or coupled to the LDH-dependent conversion of pyruvate to lactate (or vice versa) as D-lactate was shown to be ineffective.

A lactate-related increase in tumor cell motility in vitro was also shown recently by Baumann et al, where lactate promoted the migratory activity of glioma cell lines (37). With the results obtained herein changes in osmolarity could be ruled out to affect tumor cell migration in vitro. Also, our experimental design included the analysis of tumor cell migratory potential after combination of lactate exposure and inhibition of the sodium/proton antiport. This approach led to a reduced number of migrated cells in the Boyden chamber assay and thus revealed that lactate itself but not an intracellular acidification is the cause of the increased tumor cell migration. This finding is supported by new data from Yang et al, who showed that the same sodium/proton antiport inhibitor amiloride reduces the invasive ability of hepatocellular carcinoma cells without affecting cellular proliferation (38).
In an independent study, we analyzed the migratory activity of SiHa cells under physiological and high $(10 \mathrm{mM})$ glucose conditions to evaluate, if the general availability of metabolic energy resources leads to enhanced migration (unpublished data). This was not the case and similar experiments were thus not performed because a microenvironmental situation with a combination of such high glucose, absences of oxygen and high lactate production seems unrealistic for malignant cells in tumors in vivo. However, extended investigations especially with drop-out media and alterative migration and invasion assays are proposed to conclusively establish the effect of lactate on migratory activity and invasive capacity of tumor cells. Nonetheless, the present data support our initial hypothesis of the correlation between lactate content in solid tumors and their metastatic potential being at least partially due to a lactate-related induction of tumor cell migration.

Tumor tissue also contains several stromal cell types such as fibroblasts, endothelial cells and immune cells. It was beyond the scope of this study to verify the impact of lactate on the migratory activity of all of these cell types. However, because the infiltration and function of various immune cell populations in tumor spheroids was shown earlier to be impaired by an increased lactate release of the spheroids (15), 
we intended to at least include one immune cell type of interest to verify this discrepant behavior as opposed to tumor cells in our Boyden chamber assay. We chose blood monocytes, the potential precursors of tumor-associated macrophages, which are the major component of the leukocyte infiltrate in many solid tumors. Tumor-associated macrophages are often characterized by lack of immunological control combined with pro-tumoral activities (39-43). The phenomenon of a high macrophage index to correlate with tumor cell proliferation, poor prognosis and/or reduced survival of patients has for example been shown for human ductal breast carcinomas (44-48). Also, in a spontaneous, genetically engineered mouse mammary gland tumor model the recruitment of tumorassociated macrophages was found to enhance tumor vascular density and to be essential for productive metastatic growth $(49,50)$.

Scientific progress throughout almost two decades has identified tumor-associated macrophages as a source of a broad range of stimulators of tumor cell survival and proliferation as well as of diverse pro-angiogenic factors and chemoattractants $(39,51-53)$. The critical role of the tumor microenvironment and -milieu on the differentiation process of blood monocytes into a tumor-associated macrophage phenotype in tumor tissues has also been studied in some detail $(39,41,54,55)$. The chemotactic responsiveness of monocytes supposedly relates to a divergent expression of chemokines and chemokine receptors $(56,57)$. Yet, the impact of lactate in this scenario is still ambiguous. We showed, that an increase in lactate concentration inhibits monocyte migration. After supplementing the media with $20 \mathrm{mM}$ lactate, a concentration which can be frequently found in solid tumors in vivo, the migratory potential was absent for these cells. This effect could not be attributed to loss in cell survival but was correlated with a reduced inducible cytokine release and monocyte activation capacity, respectively. From the data obtained here a threshold of $10 \mathrm{mM}$ lactate for monocyte reactions might be proposed, that triggers a reduced migration and cytokine release. Only the addition of $10 \mathrm{mM}$ lactate or more induced visible changes in monocyte motility and secretion of IL-6 or TNF. Interestingly, this concentration seems to be in accordance with the threshold of $10 \mathrm{mM}$ lactate per $\mathrm{g}$ tumor tissue defined by Walenta et al, who correlated overall lactate contents in primary lesions with disease-free survival as well as the potential for metastasis (18). The phenomenon of lactate-related immune cell dysfunction clearly requires further mechanistic insight. Extended studies are thus envisioned to evaluate the impact of lactate in more detail, e.g. with and without acidosis and/or under variable oxygen concentrations, and to verify the mechanisms how lactate affects monocyte cell metabolism and in turn alters the activation process. Most recently, Dietl et al described that lactic acid plus acidification very effectively inhibit LPS-induced cytokine production in monocytes. In particular, the impact of lactate on TNF secretion was clearly concentration-dependent and much more pronounced as in the present study where the extracellular $\mathrm{pH}$ had been readjusted to 7.4. Mechanistically it could be shown, that TNF secretion by human monocytes is accompanied by increased glycolytic flux, required intact glucose utilization and export of lactate and protons which is largely abrogated in a lactate-enriched, acidic environment (58).
Interestingly, a considerable fraction of tumor-associated macrophages is located in the fibroblast- and ECM-rich stromal compartment and not directly adjacent to tumor cells in desmoplastic carcinomas. Desmoplastic fibroblasts are prominent in many aggressive tumor entities such as breast, pancreatic or squamous cell carcinomas and are therefore considered as local immune modulators in tumor tissue $(30,59,60)$. On the basis of the data presented herein one may also speculate that high lactate levels in tumor areas prevent the infiltration by blood monocytes so that the majority of this immune cell population gets stuck in the fibroblastic stromal regions. The few monocytes that manage to successfully penetrate tumor cell islets may either be immune suppressed by the milieu conditions containing lactate as indicated by the reduced inducible cytokine release after lactate exposure or they may even die as implicated by the reduced cell survival in a $40 \mathrm{mM}$ lactate environment. Such extreme concentrations are sometimes found locally restricted in high-lactate tumors. This theory is supported by recently published data from Sattler et al, who showed that tumor xenografts from head and neck tumors with high lactate content were less infiltrated with $\mathrm{CD} 45^{+}$monocytes as compared to xenografts with a low lactate content (61). Although it is difficult to simulate in vitro all aspects of highly variable in vivo tumor milieu as discussed earlier, investigations of monocytes under the influence of a lactate and $\mathrm{pH}$ gradients might be promising to further elucidate this phenomenon.

In a first attempt to characterize molecular differences between tumor cells and monocytes resulting in different migratory patterns we analyzed the expression and phosphorylation status of one important adhesion molecule. The molecular analysis of the $\beta_{1}$ integrin expression revealed an increase in protein expression after the addition of $20 \mathrm{mM}$ L-lactate but a decrease in $\beta_{1}$ integrin phosphorylation under the same conditions. The correlation of a high $\beta_{1}$ integrin expression and a metastatic phenotype was found in a variety of tumor entities, e.g. melanoma, breast or prostate (62). In addition, an inhibition of melanoma cell invasion and migration by a $\beta_{1}$ integrin specific antibody could be shown by Melchiori et al (63). These results are in accordance with our data, suggesting that the migratory pattern induced by lactate depend upon $\beta_{1}$ integrin expression but not necessarily on the phosphorylation of this protein.

We also investigated the expression and phosphorylation status of $\beta_{1}$ integrin in blood monocytes. Although donordependent differences in expression level and phosphorylation could be observed no general changes became apparent. We therefore assume that the migration and especially the lactateinduced changes in monocyte motility, as opposed to tumor cells, did not relate to $\beta_{1}$ integrin. But as $\beta_{1}$ integrin signaling is very complex, further investigations are needed, e.g. $\beta_{1}$ integrin inhibition experiments, for clarifying this relationship. Also, it has to be taken into account, that integrin expression was studied in monocyte suspension culture and non-adherent monocytes. Interestingly, in a preliminary study we observed a massive reduction of monocyte adherence to polycarbonate membrane but not culture surface when exposed to $20 \mathrm{mM}$ lactate (data not shown). This indeed indicates that a loss of specific cell adhesion features may contribute to our observation of reduced monocyte migration in the Boyden chamber 
assay towards lactate enriched media. There is clear demand for extended studies on the regulation of adherence molecules on monocytes under pathophysiologic condition and their relevance for migration.

From the present study we conclude that L-lactate promotes tumor growth and invasion by enhancing cancer cell motility and migration but impairs immune cell mobility and function. Transferring the obtained data into the clinic one might thus suggest, that high lactate levels favor tumor progression by contributing to the phenomenon of tumor immune escape in parallel to enhancing the migratory potential of the malignant cell population which may directly be coupled to lead to a higher incidence of metastasis.

\section{Acknowledgements}

This investigation was supported by the Deutsche Forschungsgemeinschaft (grant Mu576/14-1 - WMK) and the German Federal Ministry of Education and Research (BMBF, grant $01 Z Z 0502$ - LAKS). OncoRay is funded by the BMBF Center for Innovation Competence program.

\section{References}

1. Schwickert G, Walenta S, Sundfor K, Rofstad EK and MuellerKlieser W: Correlation of high lactate levels in human cervical cancer with incidence of metastasis. Cancer Res 55: 4757-4759, 1995.

2. Walenta S, Salameh A, Lyng H, Evensen JF, Mitze M, Rofstad EK and Mueller-Klieser W: Correlation of high lactate levels in head and neck tumors with incidence of metastasis. Am J Pathol 150: 409-415, 1997.

3. Brizel DM, Schroeder T, Scher RL, Walenta S, Clough RW, Dewhirst MW and Mueller-Klieser W: Elevated tumor lactate concentrations predict for an increased risk of metastases in head-and-neck cancer. Int J Radiat Oncol Biol Phys 51: 349-353, 2001.

4. Walenta S, Wetterling M, Lehrke M, Schwickert G, Sundfor K, Rofstad EK and Mueller-Klieser W: High lactate levels predict likelihood of metastases, tumor recurrence, and restricted patient survival in human cervical cancers. Cancer Res 60: 916-921, 2000

5. Walenta S, Chau TV, Schroeder T, Lehr HA, Kunz-Schughart LA, Fuerst A and Mueller-Klieser W: Metabolic classification of human rectal adenocarcinomas: a novel guideline for clinical oncologists? J Cancer Res Clin Oncol 129: 321-326, 2003

6. Walenta S, Schroeder T and Mueller-Klieser W: Metabolic mapping with bioluminescence: basic and clinical relevance. Biomol Eng 18: 249-262, 2002.

7. Bertout JA, Patel SA and Simon MC: The impact of $\mathrm{O}_{2}$ availability on human cancer. Nat Rev Cancer 8: 967-975, 2008.

8. Chen Z, Lu W, Garcia-Prieto C and Huang P: The Warburg effect and its cancer therapeutic implications. J Bioenerg Biomembr 39: 267-274, 2007.

9. DeBerardinis RJ, Sayed N, Ditsworth D and Thompson CB: Brick by brick: metabolism and tumor cell growth. Curr Opin Genet Dev 18: 54-61, 2008.

10. DeBerardinis RJ, Lum JJ, Hatzivassiliou G and Thompson CB: The biology of cancer: metabolic reprogramming fuels cell growth and proliferation. Cell Metab 7: 11-20, 2008

11. Mathupala SP, Ko YH and Pedersen PL: Hexokinase-2 bound to mitochondria: cancer's stygian link to the 'Warburg Effect' and a pivotal target for effective therapy. Semin Cancer Biol 19: 17-24, 2009.

12. Mazurek S: Pyruvate kinase type M2: a key regulator within the tumour metabolome and a tool for metabolic profiling of tumours. Ernst Schering Found Symp Proc 99-124, 2007.

13. Robey RB and Hay N: Is Akt the 'Warburg kinase'? Akt-energy metabolism interactions and oncogenesis. Semin Cancer Biol 19: 25-31, 2009.

14. Semenza GL: HIF-1 mediates the Warburg effect in clear cell renal carcinoma. J Bioenerg Biomembr 39: 231-234, 2007.
15. Gottfried E, Kunz-Schughart LA, Ebner S, Mueller-Klieser W, Hoves S, Andreesen R, Mackensen A and Kreutz M: Tumorderived lactic acid modulates dendritic cell activation and antigen expression. Blood 107: 2013-2021, 2006.

16. Fischer K, Hoffmann P, Voelkl S, Meidenbauer N, Ammer J, Edinger M, Gottfried E, Schwarz S, Rothe G, Hoves S, Renner K, Timischl B, Mackensen A, et al: Inhibitory effect of tumor cellderived lactic acid on human T cells. Blood 109: 3812-3819, 2007.

17. Walenta S, Schroeder T and Mueller-Klieser W: Lactate in solid malignant tumors: potential basis of a metabolic classification in clinical oncology. Curr Med Chem 11: 2195-2204, 2004.

18. Walenta S and Mueller-Klieser WF: Lactate: mirror and motor of tumor malignancy. Semin Radiat Oncol 14: 267-274, 2004.

19. Warburg O: On the origin of cancer cells. Science 123: 309-314, 1956.

20. Stock C and Schwab A: Protons make tumor cells move like clockwork. Pflugers Arch 458: 981-992, 2009.

21. Kroemer $\mathrm{G}$ and Pouyssegur J: Tumor cell metabolism: cancer's Achilles' heel. Cancer Cell 13: 472-482, 2008.

22. Swietach P, Vaughan-Jones RD and Harris AL: Regulation of tumor $\mathrm{pH}$ and the role of carbonic anhydrase 9. Cancer Metastasis Rev 26: 299-310, 2007.

23. Kasid U, Pfeifer A, Weichselbaum RR, Dritschilo A and Mark GE: The raf oncogene is associated with a radiationresistant human laryngeal cancer. Science 237: 1039-1041, 1987.

24. Mauceri HJ, Seetharam S, Salloum RM, Vokes EE and Weichselbaum RR: Treatment of head and neck and esophageal xenografts employing Alimta and concurrent ionizing radiation. Int J Oncol 19: 833-835, 2001.

25. Heo DS, Snyderman C, Gollin SM, Pan S, Walker E, Deka R, Barnes EL, Johnson JT, Herberman RB and Whiteside TL: Biology, cytogenetics, and sensitivity to immunological effector cells of new head and neck squamous cell carcinoma lines. Cancer Res 49: 5167-5175, 1989.

26. Friedl F, Kimura I, Osato T and Ito Y: Studies on a new human cell line $(\mathrm{SiHa})$ derived from carcinoma of uterus. I. Its establishment and morphology. Proc Soc Exp Biol Med 135: 543-545, 1970.

27. Andreesen R, Scheibenbogen C, Brugger W, Krause S, Meerpohl HG, Leser HG, Engler H and Lohr GW: Adoptive transfer of tumor cytotoxic macrophages generated in vitro from circulating blood monocytes: a new approach to cancer immunotherapy. Cancer Res 50: 7450-7456, 1990.

28. Stevenson HC, Miller P, Akiyama Y, Favilla T, Beman JA, Herberman R, Stull H, Thurman G, Maluish A and Oldham R: A system for obtaining large numbers of cryopreserved human monocytes purified by leukapheresis and counter-current centrifugation elutriation (CCE). J Immunol Methods 62: 353-363, 1983.

29. Konur A, Kreutz M, Knuchel R, Krause SW and Andreesen R: Cytokine repertoire during maturation of monocytes to macrophages within spheroids of malignant and non-malignant urothelial cell lines. Int J Cancer 78: 648-653, 1998.

30. Silzle T, Kreutz M, Dobler MA, Brockhoff G, Knuechel R and Kunz-Schughart LA: Tumor-associated fibroblasts recruit blood monocytes into tumor tissue. Eur J Immunol 33: 1311-1320, 2003.

31. Krause SW, Kreutz M and Andreesen R: Isolation, characterization and cultivation of human monocytes and macrophages. Meth Microbiol 25: 663-684, 1998.

32. Friedl P: Prespecification and plasticity: shifting mechanisms of cell migration. Curr Opin Cell Biol 16: 14-23, 2004.

33. Juel C: Intracellular $\mathrm{pH}$ recovery and lactate efflux in mouse soleus muscles stimulated in vitro: the involvement of sodium/ proton exchange and a lactate carrier. Acta Physiol Scand 132: 363-371, 1988 .

34. Hood JD and Cheresh DA: Role of integrins in cell invasion and migration. Nat Rev Cancer 2: 91-100, 2002.

35. Fujita S, Suzuki H, Kinoshita M and Hirohashi S: Inhibition of cell attachment, invasion and metastasis of human carcinoma cells by anti-integrin beta 1 subunit antibody. Jpn J Cancer Res 83: 1317-1326, 1992.

36. Wennerberg K, Fassler R, Warmegard B and Johansson S: Mutational analysis of the potential phosphorylation sites in the cytoplasmic domain of integrin beta1A. Requirement for threonines 788-789 in receptor activation. J Cell Sci 111: 1117-1126, 1998.

37. Baumann F, Leukel P, Doerfelt A, Beier CP, Dettmer K, Oefner PJ, Kastenberger M, Kreutz M, Nickl-Jockschat T, Bogdahn U, Bosserhoff AK and Hau P: Lactate promotes glioma migration by TGF-beta2-dependent regulation of matrix metalloproteinase-2. Neuro Oncol 11: 368-380, 2009. 
38. Yang X, Wang D, Dong W, Song Z and Dou K: Suppression of $\mathrm{Na}(+) / \mathrm{H}(+)$ exchanger 1 by RNA interference or amiloride inhibits human hepatoma cell line SMMC-7721 cell invasion. Med Oncol 28: 385-390, 2011.

39. Allavena P, Sica A, Solinas G, Porta C and Mantovani A: The inflammatory micro-environment in tumor progression: the role of tumor-associated macrophages. Crit Rev Oncol Hematol 66 : $1-9,2008$

40. Porta C, Subhra KB, Larghi P, Rubino L, Mancino A and Sica A Tumor promotion by tumor-associated macrophages. Adv Exp Med Biol 604: 67-86, 2007.

41. Mantovani A, Schioppa T, Porta C, Allavena P and Sica A: Role of tumor-associated macrophages in tumor progression and invasion. Cancer Metastasis Rev 25: 315-322, 2006.

42. al-Sarireh B and Eremin O: Tumour-associated macrophages (TAMS): disordered function, immune suppression and progressive tumour growth. J R Coll Surg Edinb 45: 1-16, 2000.

43. Mantovani A, Bottazzi B, Colotta F, Sozzani S and Ruco L: The origin and function of tumor-associated macrophages. Immunol Today 13: 265-270, 1992.

44. Tsutsui S, Yasuda K, Suzuki K, Tahara K, Higashi H and Era S: Macrophage infiltration and its prognostic implications in breast cancer: the relationship with VEGF expression and microvessel density. Oncol Rep 14: 425-431, 2005.

45. Bingle L, Brown NJ and Lewis CE: The role of tumourassociated macrophages in tumour progression: implications for new anticancer therapies. J Pathol 196: 254-265, 2002.

46. Leek RD, Hunt NC, Landers RJ, Lewis CE, Royds JA and Harris AL: Macrophage infiltration is associated with VEGF and EGFR expression in breast cancer. J Pathol 190: 430-436, 2000.

47. Goede V, Brogelli L, Ziche M and Augustin HG: Induction of inflammatory angiogenesis by monocyte chemoattractant protein-1. Int J Cancer 82: 765-770, 1999.

48. Leek RD, Lewis CE, Whitehouse R, Greenall M, Clarke J and Harris AL: Association of macrophage infiltration with angiogenesis and prognosis in invasive breast carcinoma. Cancer Res 56 : 4625-4629, 1996.

49. Lin EY, Nguyen AV, Russell RG and Pollard JW: Colonystimulating factor 1 promotes progression of mammary tumors to malignancy. J Exp Med 193: 727-740, 2001.

50. Lin EY, Li JF, Gnatovskiy L, Deng Y, Zhu L, Grzesik DA, Qian H, Xue XN and Pollard JW: Macrophages regulate the angiogenic switch in a mouse model of breast cancer. Cancer Res 66: 11238-11246, 2006.

51. Coffelt SB, Hughes R and Lewis CE: Tumor-associated macrophages: effectors of angiogenesis and tumor progression. Biochim Biophys Acta 1796: 11-18, 2009.
52. Lewis CE and Pollard JW: Distinct role of macrophages in different tumor microenvironments. Cancer Res 66: 605-612, 2006.

53. Goswami S, Sahai E, Wyckoff JB, Cammer M, Cox D, Pixley FJ, Stanley ER, Segall JE and Condeelis JS: Macrophages promote the invasion of breast carcinoma cells via a colony-stimulating factor-1/epidermal growth factor paracrine loop. Cancer Res 65: 5278-5283, 2005.

54. Lewis CE and Hughes R: Inflammation and breast cancer. Microenvironmental factors regulating macrophage function in breast tumours: hypoxia and angiopoietin- 2 . Breast Cancer Res 9: 209, 2007.

55. Crowther M,Brown NJ, Bishop ET and Lewis CE: Microenvironmental influence on macrophage regulation of angiogenesis in wounds and malignant tumors. J Leukoc Biol 70: 478-490, 2001.

56. Soria $G$ and Ben-Baruch A: The inflammatory chemokines CCL2 and CCL5 in breast cancer. Cancer Lett 267: 271-285, 2008.

57. Weber C, Belge KU, von HP, Draude G, Steppich B, Mack M, Frankenberger M, Weber KS and Ziegler-Heitbrock HW: Differential chemokine receptor expression and function in human monocyte subpopulations. J Leukoc Biol 67: 699-704, 2000.

58. Dietl K, Renner K, Dettmer K, Timischl B, Eberhart K, Dorn C, Hellerbrand C, Kastenberger M, Kunz-Schughart LA, Oefner PJ Andreesen R, Gottfried E and Kreutz MP: Lactic acid and acidification inhibit TNF secretion and glycolysis of human monocytes. J Immunol 184: 1200-1209, 2010.

59. Silzle T, Randolph GJ, Kreutz M and Kunz-Schughart LA: The fibroblast: sentinel cell and local immune modulator in tumor tissue. Int J Cancer 108: 173-180, 2004.

60. Kunz-Schughart LA and Knuechel R: Tumor-associated fibroblasts (part II): Functional impact on tumor tissue. Histol Histopathol 17: 623-637, 2002.

61. Sattler UG, Meyer SS, Quennet V, Hoerner C, Knoerzer H, Fabian C, Yaromina A, Zips D, Walenta S, Baumann M and Mueller-Klieser W: Glycolytic metabolism and tumour response to fractionated irradiation. Radiother Oncol 94: 102-109, 2010.

62. Felding-Habermann B: Integrin adhesion receptors in tumor metastasis. Clin Exp Metastasis 20: 203-213, 2003.

63. Melchiori A, Mortarini R, Carlone S, Marchisio PC, Anichini A, Noonan DM and Albini A: The alpha 3 beta 1 integrin is involved in melanoma cell migration and invasion. Exp Cell Res 219: 233-242, 1995 . 